\title{
The ARM Network - a model for infectious disease surge response capacity in the Western Pacific Region
}

\author{
Shoaib Hassan, a Martyn Kirk, ${ }^{b}$ Tony Stewart, ${ }^{c}$ and C. Raina Maclntyre ${ }^{a}$ \\ Correspondence to Raina MacIntyre (e-mail: r.macintyre@unsw.edu.au).
}

$\mathrm{T}$ he United States Centers for Disease Control and Prevention (US CDC) through its Epidemic Intelligence Service (EIS) programme provides a model for field epidemiology training programmes (FETPs) and has spawned FETPs worldwide. ${ }^{1}$ The unique training provided by FETPs equips graduates to respond to public health emergencies in the field, including establishing surveillance and investigating outbreaks of disease. The EIS and other FETPs have well-established networks of alumni that provide capacity for responding to public health disasters.

In 1989, the Commonwealth Government of Australia funded two initiatives, the Communicable Diseases Network of Australia (which has an advisory and national communication and coordination function rather than an operational response function) and the National Centre of Epidemiology and Population Health (NCEPH) at ANU. Australia's FETP based at the Australian National University (ANU) was established in 1991. The two-year research programme, based on the EIS model, leads to a Masters qualification in Applied Epidemiology (MAE). Historically, the MAE programme has provided surge capacity through FETP trainees for national and international infectious diseases outbreaks and emergencies, but with only around 20 trainees at any one time, the scope of this capacity is limited. Response to international events focuses on the World Health Organization (WHO) Western Pacific Region where many countries do not have their own FETPs. The Western Pacific Region includes approximately 50\% of the world's population. The Region also has difficultto-access small island countries with populations spread over large distances. Some of these countries have national organizations, institutes and FETPs, while other small countries rely on international public health support. ${ }^{2}$ The Region suffers a disproportionate burden of disease from preventable infections, and has variable response capacity. Infectious diseases such as measles, vector-borne diseases and cholera have a potential to spread rapidly and are a challenge in the Region. ${ }^{3,4}$

\section{Natural disasters and the regional response capacity}

Over the last decade, the Western Pacific Region has faced various natural disasters resulting in public health emergencies affecting both developed and developing countries. Such events have ranged from earthquakes in New Zealand to a typhoon in the Philippines and a nuclear disaster in Japan. ${ }^{5}$ The Solomon Islands recently had earthquakes and flash floods that had a major impact on public health systems. ${ }^{5}$

Such emergencies are often beyond the state and national governments' capacities, and regional or crossjurisdictional responses are required. Management of these events ranges from preparedness to acute-phase response and recovery, all of which demand financial and technical commitments. Natural disasters can turn into complex emergencies, especially in the presence of a pre-existing or growing burden of communicable and noncommunicable diseases. Risk mitigation and preparedness for such challenges at national levels can be achieved by regional efforts. ${ }^{6,7}$ Public health emergencies due to an outbreak or natural disaster may cross national borders and even spread to other regions in a short period of time. No country is free from

School of Public Health and Community Medicine, University of New South Wales, Sydney, New South Wales, Australia.

National Centre for Epidemiology and Population Health, The Australian National University, Canberra, Australian Capital Territory, Australia.

Burnet Institute, Melbourne Victoria, Australia.

Submitted: 27 May 2014; Published: 16 June 2014

doi: 10.5365/wpsar.2014.5.2.011 
such risks, but countries without FETPs may be more vulnerable. Australia, as a high-income country with a long-established, high-quality FETP, has the skills and capacity to provide assistance in the Region.

\section{Australian response capacity}

Australia is a federation of six states and two territories, with national expert committees in infectious diseases and a national incident room but no equivalent of the US CDC or national field response capacity. To strengthen response to emerging infectious disease threats in the Asia Pacific region, the AusReady Facility was funded by the Australian Agency for International Development from 2006 to 2010. The facility was tasked to manage a database of experts and focus on outbreak prevention and preparedness, but it did not establish extensive partnerships with other networks, had a relatively low number of deployments and lacked ongoing funding. ${ }^{8}$

The states of New South Wales and Victoria have had public health officer training programmes. Currently, only the New South Wales programme remains. These programmes, while loosely based on the EIS model, offer broad-based public health and policy training but are not focused on infectious diseases field epidemiology. ${ }^{9}$ Further, state-based responses to local outbreaks are constrained within state boundaries, and rarely deploy staff for international response. The MAE programme has produced over 160 trained field epidemiologists, participated in over 300 national and international outbreak responses and established or evaluated a variety of surveillance systems over the past 25 years. ${ }^{10}$ The MAE programme is working well, with eight to 10 scholars being recruited each year into field placements, and it continues to provide some surge capacity to Australian and regional governments. Other than the limited capacity provided by the MAE programme, there is no national mechanism to harness and deploy Australia's skilled public health workers for international response.

\section{Australian Response MAE Network}

With many skilled public health professionals and a highquality FETP, Australia is ideally placed to contribute to the control of infectious diseases regionally. While Australia has response capacity for trauma and emergencies, there was no nationally funded mechanism for deploying qualified professionals for infectious diseases outbreak responses that cross national and international borders. In May 2012, at a national forum on field epidemiology at the University of New South Wales (UNSW), a critical gap in national and international field epidemiology response capacity was identified. ${ }^{11}$ The momentum set at this meeting, with continued engagement of interested stakeholders into 2013, led to the genesis of the Australian Response MAE (ARM) Network.

The ARM Network was established by three MAE alumni to address this gap and to support Australia's regional responsibility and role in assisting in public health emergencies. ${ }^{12}$ The ARM Network was founded by ANU, Burnet Institute and UNSW to identify experienced Australian public health professionals with skills in field epidemiology, applied public health and emergency response. ${ }^{12}$ All three founding partner institutions are members of WHO's Global Outbreak Alert and Response Network (GOARN) and receive alerts and requests for assistance. ARM partners maintain their own networks of public health professionals, including students and graduates of FETPs or other relevant programmes, to provide surge capacity when required. Other suitably skilled public health professionals may apply to join ARM Network; there has been a high level of interest, and over 50 new members from around Australia have joined in the six months since the ARM Network was established.

The ARM Network was first used in response to Typhoon Haiyan in the Philippines in November 2013. Through the ARM Network, at least five field epidemiologists were deployed to assist with response to the public health emergency through GOARN. The ARM Network uses an operational model to identify, screen and mobilize experienced public health professionals with skills in field epidemiology, applied public health and outbreak response in the event of global, regional or cross-jurisdictional public health emergencies (Figure 1). ARM members are connected through a private online network where deployment opportunities and other resources are posted. This network also allows discussion and feedback following deployment. ARM Network works with partners such as GOARN and Registered Engineers for 
Figure 1. ARM Network operational model for assessment and deployment of public health professionals

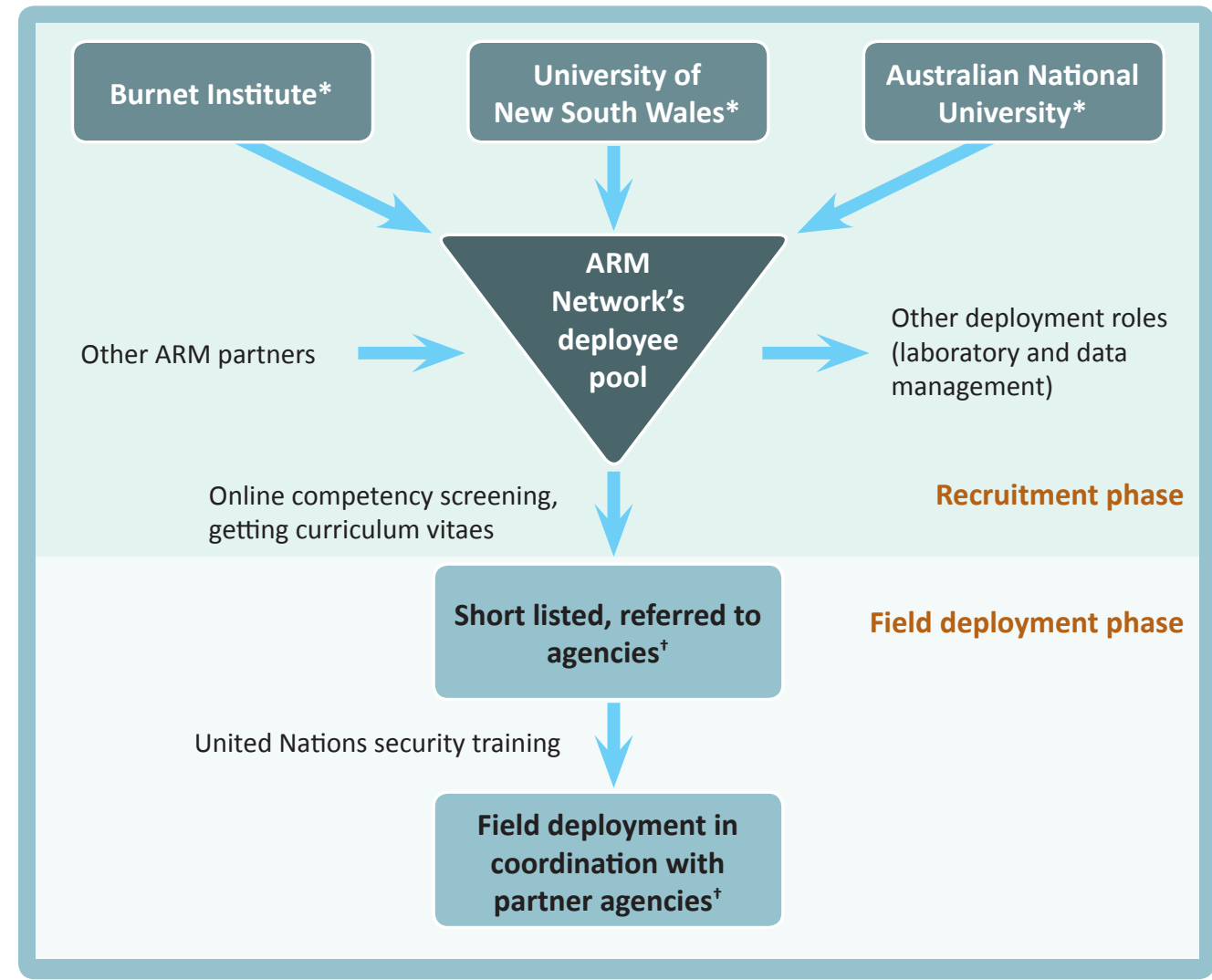

ARM - Australian Response MAE (Master of Applied Epidemiology)

* Founding partners of the ARM Network.

† Agencies include World Health Organization, Global Outbreak Alert and Response Network (GOARN), Registered Engineers for Disaster Relief (RedR), Australian Medical Assistance Teams (AUSMAT) and other potential partners.

Disaster Relief (RedR). ARM Network provides a focal point for Australian infectious diseases surge response capacity.

The ARM Network operates with the in-kind support of three institutions, linked by the common thread of FETP training, which recognizes the important contribution of field epidemiology to national and international response capacity. Stakeholders in the national and international public health community have been made aware of the ARM Network that is being formally launched on 16 June $2014 .{ }^{12}$ To widen the engagement and awareness, a public website accepts requests from anyone with relevant skills to join ARM Network. ${ }^{12}$ When ARM Network receives a request for assistance, a senior network member is assigned as the contact for the requesting agency. A call for assistance is sent to ARM Network members through the private members online network. Suitable candidates applying for field deployment are then referred to a partner agency such as GOARN, Australian Medical Assistance Teams or RedR for deployment. ARM Network will evaluate the usefulness of deployment to ensure continuous feedback and improvement.

\section{Way forward}

ARM Network offers an organizational model for FETPs and alumni in the Region to assist with public health and infectious diseases emergencies. Our experience has been that there is a large body of skilled professionals who are willing to contribute to surge response capacity, and ARM Network provides a mechanism for them to do so. The network's operational model has the capacity to grow and the scope may broaden over time. ARM Network provides the Western Pacific Region with 
skilled professionals who can support management and control of infectious diseases during public health and civil emergencies.

\section{Funding}

The ARM Network initiative is funded by in-kind support from the School of Public Health and Community Medicine, UNSW, the Burnet Institute and NCEPH, ANU.

\section{Conflicts of interest}

The authors are the founders of ARM Network.

\section{Acknowledgements}

The University of New South Wales (UNSW), the Burnet Institute and the Australian National University (ANU) are acknowledged for the in-kind support of ARM Network.

\section{References:}

1. Schneider D et al. Training the global public health workforce through applied rpidemiology training programs: CDC's Experience, 1951-2011. Public Health Reviews, 2011, 33(1):190203.

2. Blakely $\mathrm{T}$ et al. Health status and epidemiological capacity and prospects: WHO Western Pacific Region. International Journal of Epidemiology, 2011, 40:1109-1121. doi:10.1093/ije/dyr014 pmid:21343183
3. Numazaki K. Current problems of measles control in Japan and Western Pacific Region. Vaccine, 2007, 25:3101-3104. doi:10.1016/j.vaccine.2007.01.105 pmid:17368662

4. Calain $P$ et al. Can oral cholera vaccination play a role in controlling a cholera outbreak? Vaccine, 2004, 22:2444-2451. doi:10.1016/j.vaccine.2003.11.070 pmid:15193408

5. Emergencies and Disasters. Manila, World Health Organization Regional Office for the Western Pacific, 2011.

6. Li A, Kasai T. The Asia Pacific Strategy for Emerging Diseases - a strategy for regional health security. Western Pacific Surveillance and Response Journal, 2011, 2:6-9. doi:10.5365/ wpsar.2011.2.1.001 pmid:23908877

7. Cuboni $\mathrm{G}$ et al. Human resources for public health challenges in the Western Pacific: local community colleges respond. Pacific Health Dialog, 2010, 16(1):173-179. pmid:20968251

8. 'AusReady' The Asia Pacific Emerging Infectious Diseases Facility - Mid-Term Review. Canberra,Department of Foreign Affairs and Trade, 2008.

9. Macintyre CR. Public health and health reform in Australia. The Medical Journal of Australia, 2011, 194:38-40. pmid:21449867

10. Master of Philosophy (Applied Epidemiology). Canberra, Australian National University, 2014 (http://nceph.anu.edu.au/education/ research-degree/master-philosophy-applied-epidemiology, accessed 29 March 2014).

11. How well could Australia respond to a public health emergency? Sydney, University of New South Wales, 2012 (http://newsroom. unsw.edu.au/news/health/how-well-could-australia-respondpublic-health-emergency, accessed 29 March 2014).

12. The Australian Response Master of Applied Epidemiology (ARM). Canberra, 2014 (http://www.arm.org.au/, accessed 29 March 2014). 